Kennesaw State University

DigitalCommons@Kennesaw State University

Faculty Publications

$12-22-2018$

\title{
Virtual Reality as a Pedagogical Tool to Design for Social Impact: a Design Case
}

\section{Tiffany Roman}

troman5@kennesaw.edu

Jon Racek

Indiana University - Bloomington, jonracek@indiana.edu

Follow this and additional works at: https://digitalcommons.kennesaw.edu/facpubs

Part of the Architectural Technology Commons, Educational Methods Commons, Educational Technology Commons, and the Scholarship of Teaching and Learning Commons

\section{Recommended Citation}

Roman, Tiffany and Racek, Jon, "Virtual Reality as a Pedagogical Tool to Design for Social Impact: a Design Case" (2018). Faculty Publications. 4819.

https://digitalcommons.kennesaw.edu/facpubs/4819

This Article is brought to you for free and open access by DigitalCommons@Kennesaw State University. It has been accepted for inclusion in Faculty Publications by an authorized administrator of DigitalCommons@Kennesaw State University. For more information, please contact digitalcommons@kennesaw.edu. 


\title{
Virtual Reality as a Pedagogical Tool to Design for Social Impact: a Design Case
}

\author{
Tiffany A. Roman ${ }^{1}$ (D) Jon Racek ${ }^{2}$ \\ Published online: 22 December 2018 \\ (C) Association for Educational Communications \& Technology 2018
}

\begin{abstract}
Three-dimensional (3-D) virtual environments have key affordances that can improve learning, particularly when context, culture, and pedagogical aims are aligned to a given learning situation. One challenge in detailing effective uses of 3-D virtual environments in teaching and learning contexts is that the design judgments involved are not always made explicit. We argue that the transparency of design judgments, as it relates to the use of 3-D virtual environments, are critically important. This article advances scholarship of emerging technologies by detailing the design judgments of a university instructor within a Design for Social Impact cross-disciplinary course. To address learner needs and the cultural aims of an authentic client-based project, the instructor directed students to sketch design ideas within Google Blocks, which allows users to create 3-D models in virtual reality. This design case provides precedent for practitioners interested in how 3-D virtual environments can align to learning contexts, cultures, and pedagogical aims.
\end{abstract}

Keywords Virtual reality $\cdot$ Emerging technologies $\cdot$ Pedagogy $\cdot$ Culture $\cdot$ Design $\cdot$ Authentic learning $\cdot$ Instructional design $\cdot$ Social impact

It has been argued that three-dimensional (3-D) virtual environments (VE) have key affordances that can improve learning. The learning benefits that stem from 3-D VE include spatial knowledge representation, experiential learning, engagement, contextual learning and collaborative learning (Dalgarno and Lee 2010); however, these stated learning benefits are often driven by the technological affordances of virtual reality rather than pedagogical requirements of a given learning experience (Fowler 2015). Recently, Fowler (2015) has argued there is a need to shift from scholarship that "starts with an analysis of the technology then seeks to derive learning benefits" (p. 420). Fowler posited that pedagogical

Tiffany A. Roman

tiffany.roman@kennesaw.edu

Jon Racek

jonracek@indiana.edu

1 Department of Instructional Technology, Kennesaw State University, Kennesaw Hall Room 2329, MD 0127, 585 Cobb Avenue, Kennesaw, GA 30144, USA

2 Indiana University, The Comprehensive Design Area, Kirkwood Hall, Room 204, 130 S Woodlawn Ave, Bloomington, IN 47405, USA decisions, including intended learning outcomes, learning contexts, and cultural issues in learning, should inform how task affordances are matched with learning requirements with respect to 3-D virtual learning environments (VLEs).

In order to advance pedagogical theory and practice with respect to 3-D VLEs, Fowler (2015) presented an expanded "design for learning" approach (see Fig. 1), which he described as:

A process that goes from a general contextual description of the teaching and learning environment through a set of teaching and learning requirements based on defining what stage the learner is at and what learning outcomes have to be achieved by undertaking a given set of learning activities. The practitioner then has to determine a particular teaching and learning approach that can best meet the requirements. The whole process can then be recorded in a learning specification. Exactly how a learning specification is presented will vary. (p. 419-420).

Fowler (2015) acknowledged that his expanded framework best aids practitioners when the principles and practices described within are presented in a way that can be 
Fig. 1 A design for learning approach. Adapted from "Virtual reality and learning: Where is the pedagogy?" by Fowler 2015, British Journal of Educational Technology, 46, p. 420. Copyright 2015 John Wiley and Sons

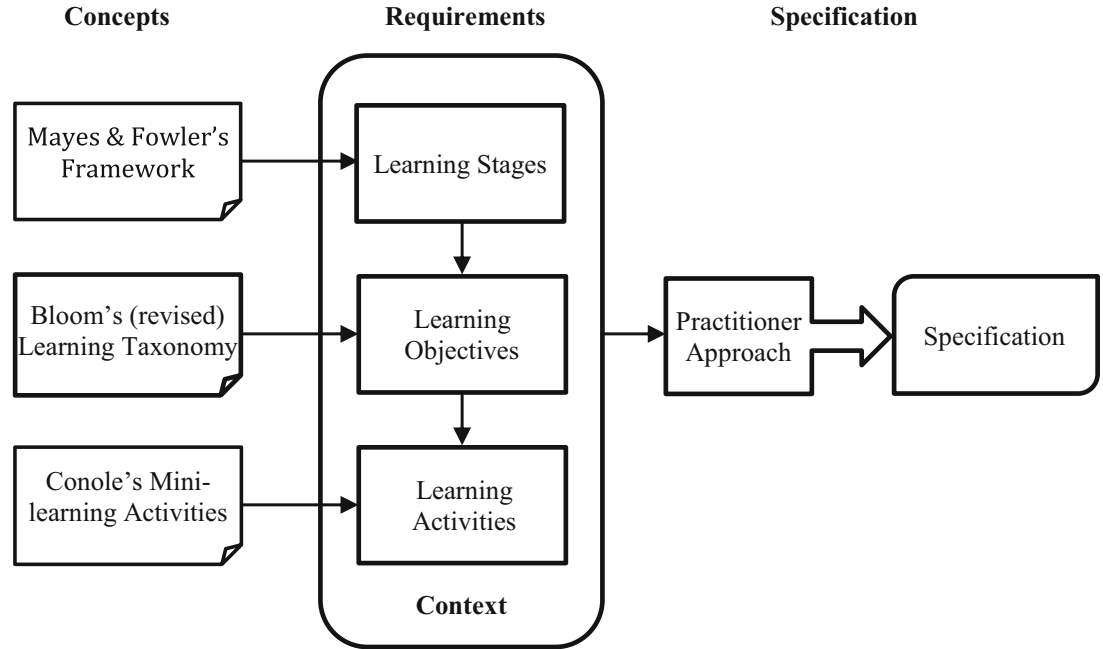

understood and applied to teaching and learning contexts. To that end, we present the integration of Fowler's expanded 3-D VLE approach in the context of a design case. A design case is a rich description "of a real artifact or experience that has been intentionally designed" (Boling 2010 , p. 1). The intent of a design case is to provide a precedent that designers can use to make similar design decisions, avoid design decisions, or select alternative options based on lessons learned through exposure to the case (Howard et al. 2012). The context of the design case we present within this article is situated within a crossdisciplinary Design for Social Impact course taught at a large Midwestern university in fall 2017.

Although Fowler's (2015) expanded 3-D VLE approach asks practitioners to consider the alignment of context, culture, and pedagogical aims to a given learning situation, we argue that the approach, as it exists currently, does not address the design judgment required on the part of the practitioner. Practitioners are required to choose and adapt methods or combinations of methods that are situational and that may each be carried out in multiple ways (Reigeluth 1999). Since Fowler posited that practitioners determine pedagogical approaches that lead to learning specifications, we argue that one's approach can only be identified when the design judgments of the practitioner are made transparent (see Boling et al. 2017). This design case advances scholarship in this area by making explicit the design judgments of a university instructor who intentionally integrated student learning activities within 3-D VLE based on the context, culture, and pedagogical aims of his course.

In this article, we (a) describe effective uses of 3-D VLEs in everyday teaching and learning, (b) demonstrate how the "designing for learning" approach proposed by Fowler (2015) can be applied in cross-disciplinary contexts, and (c) highlight how culture can be considered throughout the design process.

\section{Instructional Context}

The Design for Social Impact course that integrated Fowler's (2015) 3-D VLE design for learning approach grew out of a university initiative intended to improve and enrich the health, prosperity, and vitality of nearby communities. Each academic year, the university selects one county as a regional focus. The university then works with community leaders to identify the region's needs. These efforts, in turn, provide high-impact teaching and learning opportunities for university faculty, staff, and students. The civic engagement and economic development projects that emerge are intended to be long-term, sustained partnerships.

In spring 2017, to address the needs of two small neighboring cities with rich cultural histories, two professors within the university's Graphic Design and Interior Design programs jointly proposed Design for Social Impact. The cities, one with a population of 13,413 and the other 4350 , asked for assistance in creating city branding and designing roadside markers at the borders of each city. Twenty-five undergraduate students, from varying disciplines, enrolled in the course. The students differed in their levels of technology skills and design knowledge, as most were non-design majors.

Throughout the design process, attention was given to the regional cultures of the cities, as recommended by Asino et al. (2017), and Fowler (2015). At the onset of the project, city leaders came to the university and provided presentations on their respective cities to the course participants. The upbeat discussions focused on the histories of the cities, famous former residents, annual festivals, iconic symbols, current projects, grant awards, and recent 
renovations. Students were provided with marketing materials as an additional resource.

To better understand the cities' unique cultures, students completed multiple field trips and were able to meet city officials, take tours of the surrounding areas, and identify notable sites (e.g., town hall, historical landmarks). To better understand the project's design materials, students toured a limestone mill centrally located to both towns. City officials also showed the site locations where the roadside markers were to be built. Students focused on understanding the values of the different cities and the image officials wanted to project.

\section{Pedagogical Decisions}

The Design for Social Impact course was divided into two 8week sessions. The first 8 weeks of the course focused on city branding and was led by the Graphic Design faculty member; the second 8 weeks was taught by the Interior Design faculty member who facilitated student designs of roadside markers. The integration of VR design activities for pedagogical purposes took place during the course's second half and is the primary focus of this design case (Boling 2010). Classroom instruction for both sessions occurred in two different spaces - a traditional computer lab and a VR-equipped active learning classroom (see Fig. 2). Active learning classrooms can vary in design and configuration; however, they are typified by movable furniture for collaborative purposes, highly accessible whiteboard space, and technology enhancements (Baepler et al. 2016).

\section{3-D Ideation within Google Blocks}

Rather than follow a traditional pattern of envisioning, hand sketching, and model building (Constable 1994; Egan 1999), the Interior Design faculty member in this design case asked his Design for Social Impact class to use the intuitive nature of VR technology to create 3-D "sketches" at the onset of their

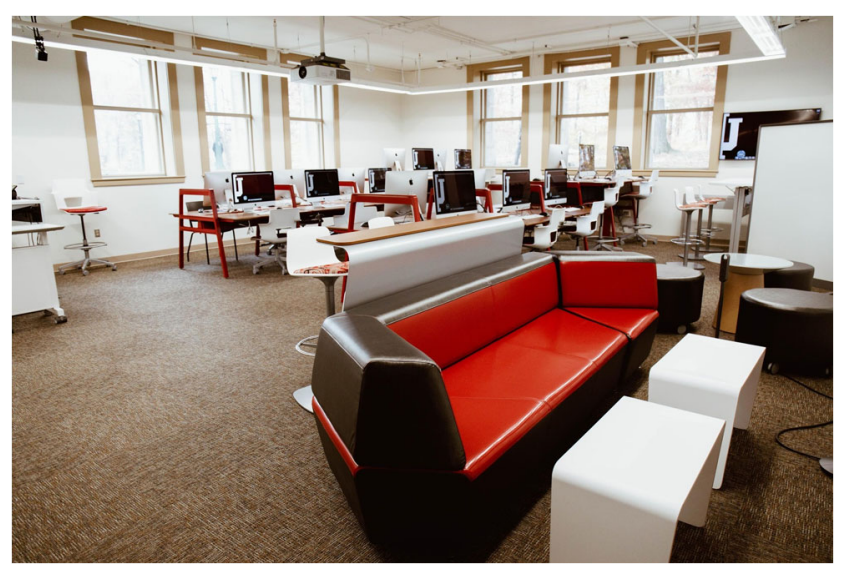

Fig. 2 The active learning classroom which features a permanent VR station eight-week project. Jumping straight into VR may seem counter intuitive; however, modeling before sketching can enhance visualization of solutions and improve student understanding of prototypes without a loss of time (Lemons et al. 2010). The instructor selected Google Blocks, a VR modeling tool launched to the public in July 2017. Designed as a free app for specific VR headsets (i.e., HTC Vive, Oculus Rift), the user interface requires the use of motion-sensing controllers. In other words, to make 3D sketches within Google Blocks, one must wear an HTC Vive or an Oculus VR headset and design using the VR controllers.

Google Blocks, as a pedagogical tool, offers several affordances. The instructor was drawn to Google Blocks because it enabled students with no previous VR experience to create 3-D models quickly. Before the existence of Google Blocks, 3-D VR modeling was extremely time-intensive; to build out a landscape within a virtual environment, users had to create new objects from scratch. Google Blocks simplified the creation process by eliminating curved surfaces. All objects rendered in Google Blocks have a low-polygon count, meaning that when one creates a sphere in the application, the aesthetic is more akin to a disco ball. By reducing the polygon count, 3-D models can then also be rendered in cheaper, phone-enabled headsets like Google's Daydream View or Samsung's Gear VR. Once users create a 3-D model, the file can easily be exported, published online for viewing, downloaded as an .obj file, or modified by other users in Google Blocks or a different 3-D VR modeling program.

In addition to a low-polygon count, the simplicity of the user interface makes Google Blocks highly approachable. Google Blocks contains only six tools: shape, stroke, paint, modify, grab, and erase. As an example of how the program works in practice, if a user wanted to make a pile of cooked spaghetti, the individual would start by creating a long thin rectangular shape. Once the shape was made, the user would use the modify tool to bend it into a "noodle" appearance. If desired, the "noodle" could be easily colored to a light-yellow hue using the paint function. To create an entire pile of spaghetti, the finished "noodle" could be copied and modified until the desired look was achieved. Google Blocks saves users' time by eliminating the need to consider lighting, shading, texture, and reflections during modeling efforts.

\section{Scaffolding Instruction within Google Blocks}

For the instructor in this design case, teaching students to sketch within Google Blocks required several instructional scaffolds to orient students to the application. On the day Google Blocks was introduced, the instructor provided a live demonstration of how to use the tool. Using the affordances of the active learning classroom, the instructor projected what he was viewing within his HTC Vive headset to a large dropdown screen in the room, enabling all the students in the course to see what he was experiencing in Google 
Blocks. Following the instructor demonstration, students received an instructor-created reference manual for the application (see Fig. 3) as Google Blocks documentation was limited at that time. Students used in-class worktime to complete a tutorial provided within the Google Blocks application. When Google Blocks first launched, as a means of exploring the tools within the program, the user tutorial involved adding additional scoops of ice cream and a cherry to an existing ice cream cone, coloring a cherry, replicating the entire cone, and eliminating the cherry in the copied version. After the completion of the tutorial, students were able to play with and explore the application without the expectation of a submission requirement.

To further develop their VR modeling skills, students practiced with Google Blocks outside of class. With the ability for students to access campus VR labs equipped with HTC Vive headsets and VR motion controllers, the instructor asked students to complete a robot-modeling exercise for homework (see http://bit.ly/bots-blocks to view the robot creation challenge that incorporated Google Blocks). The robot creation exercise enabled students to explore an exemplary use of the Google Blocks as a tool, as all components of the robot were previously modeled. The exercise was free to download online, making it readily accessible. Additionally, the exercise afforded students the opportunity to have additional practice with the tool in general, which the instructor hoped would ultimately reduce potential frustration with the interface. Despite good pedagogical intentions, students encountered challenges in carrying out the robot exercise. The instructor did not anticipate the substantial amount of geometry involved nor the sheer number of parts/subparts available to students, which proved to be overwhelming. Additionally, time was a factor in end product results; some students encountered lag time in using the program whereas others spent too much time making their creations.
After the completions of the introductory exercise, students used HTC Vive headsets, VR motion-controllers, and Google Blocks to create initial 3-D sketches of their roadside marker prototypes, which were later refined in Google Sketchup and Adobe Illustrator. Following the advice of Dalsgaard (2017) regarding the role of tools within the design process, the instructor sought to develop students' skills with a specific technology (i.e., Google Blocks) in order to reach a desired outcome (i.e., simplified sketch of a roadside marker). At the same time, the instructor facilitated student understanding of the roles, potentials, and limitations of the other tools (i.e., Sketchup, Illustrator) that were applied later within the project. In this first phase of designing, students created three roadside markers for each town for a sum of six total models rendered. As articulated earlier, the decision to have students design within a 3-D VLE at the onset of the project was driven by the fact that the course itself was targeted to non-designers who lacked prior experience with industry standard design software. Instead of teaching a program like Sketchup - a relatively complex visualization tool-for several weeks, the low threshold to entry and intuitive nature of the VR Google Blocks allowed students to immediately jump into designing and creating 3-D sketches.

To support students' modeling efforts within Google Blocks, the course instructor provided students with a "kit of parts" to help facilitate ideation. The kit of parts included agreed-upon city symbols determined by the class. The parts within the kit were either created by the instructor for the students or the renderings were found online. The intent of the parts kit was to allow students to start building with support, as opposed to beginning with a blank canvas. The kit of parts, however, proved to be problematic as some students created prototypes of the roadside markers that relied heavily on the provided prefabricated components. Students found it difficult to disassociate from their previous branding efforts and to move beyond the symbols for which the cities were known. For
Fig. 3 Excerpt from the instructor-created Google Blocks' instruction manual

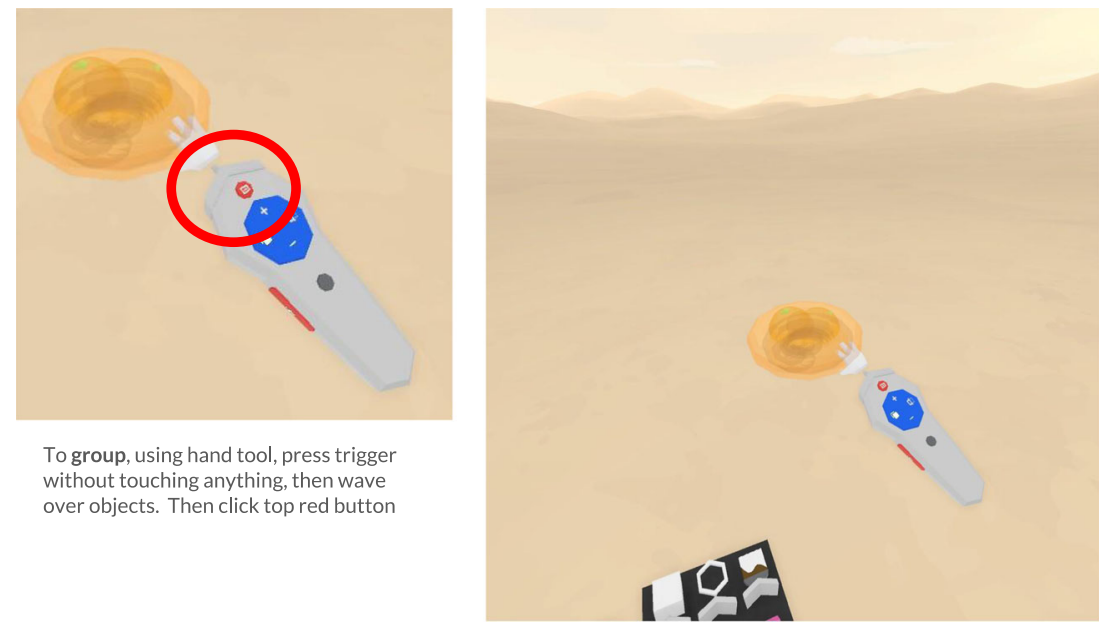


example, some students took a "fruit salad" approach by combining multiple city symbols into their designs (see Fig. 4).

\section{Challenges and Benefits of 3-D Sketches}

Prototyping ideas within Google Blocks proved to have both challenges and benefits. In reflecting on the experience of the initial prototype designs, the instructor cited several lessons learned. In hindsight, he regretted providing the kit of parts to his students for ideation, due to the degree to which some students relied on it. Despite that particular setback, the instructor thought that integrating Google Blocks from the beginning worked well due to the tool's simplicity and its quick 3-D sketching capabilities. The instructor noted that students had the ability to explore the geometric design capabilities within the application and, as a result, were able to create prototypes with strong aesthetic qualities, such as the example highlighted in Fig. 5.

While acknowledging these benefits, Google Blocks could also be difficult to control. For example, when lines are joined, sometimes they may appear offset rather than seamless. This glitch resulted in a certain level of dissatisfaction for students who desired more finished or accurate representations. Once the instructor framed the intent of the tool as a "3-D sketch" application, students were more willing to accept the limitations of the product.

As an alternate approach to Google Blocks, the instructor considered using Gravity Sketch (gravitysketch.com), a professional schematic design tool. He explained to his students that, in the past, professional designers would have started their work by hand sketching. With Gravity Sketch, professional designers can create 3-D models quickly within an immersive setting, extract the models, and import them directly into another 3-D creation tool (e.g., Rhino) to further enhance dimensional accuracy. The instructor shared that

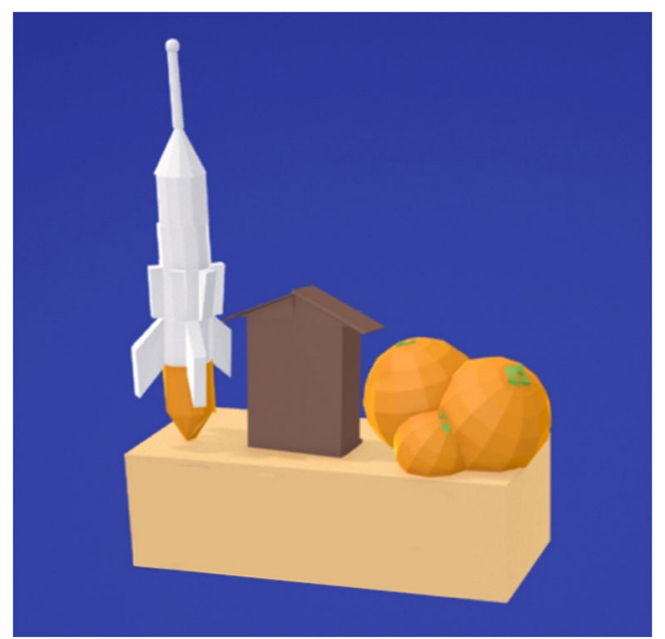

Fig. 4 An example of an initial prototype in Google Blocks that incorporated multiple symbols into the design

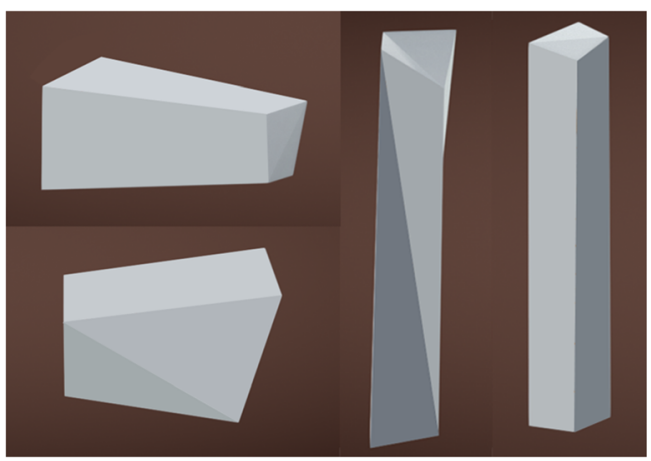

Fig. 5 Example of initial prototypes that explored the geometric design capabilities of Google Blocks

professional designers might incorporate multiple VR modeling tools (e.g., Google Blocks, Gravity Sketch, Rhino, CAD) into their project workflow, an approach he presented with advanced interior design students. For the purposes of the Design for Social Impact course, however, Google Blocks served the instructor's objectives best.

\section{The Role of Critique, Reflection, and Revisions within 3-D VLEs}

An integral component of the revision process for students included small group critiques. After students created their six initial prototypes within Google Blocks, rather than facilitate a large class critique, the instructor intentionally divided students into small groups (i.e., four to five individuals). Small group critiques were spread out between the active learning classroom and a classroom located across the hall. Students presented their roadside marker prototypes by using Mersive Solstice in conjunction with the large flat-screen displays/inroom projectors in the classrooms. Mersive Solstice enables the sharing of content from any laptop or mobile device to an in-room display. The instructor floated between groups providing constructive feedback during the hour-long review session. In order for students to build trust and comfort with their peers, the group configurations remained constant during the eight-week project.

Following small group critiques, on a separate date, the instructor held personalized student-instructor feedback sessions. The instructor met with two students at a time; each student was allotted a one-on-one conversation that lasted about 7 to 8 minutes. The instructor verbally reviewed the roadside marker prototype designs with the student designer while the individual viewed her work within Google Blocks using the HTC Vive headset and VR motion controllers. Students had the opportunity to listen to the instructor's feedback while immersed in the 3-D VLE as the partnering student took notes on the student designer's behalf. The feedback sessions also enabled students to mention and solve any issues that they were having with the VR technologies. 
From the instructor's perspective, the 3-D VLE feedback sessions enabled rich discussions about students' VR project work. The ideas behind the 3-D prototypes and the ways in which the designs aligned with the cultures of the cities proved to be the most common topic of discussion across all instructor-student feedback sessions. The instructor also reported that a key benefit of holding the critiques within a 3D VLE was the ability to talk about scale in a tangible manner. For example, if students created their design at a "model scale," the instructor encouraged students to modify their work to reflect a full-scale prototype. He also asked students to consider using "people" as a reference point within their 3D VLE by creating a block that was six-feet tall and incorporating it into their design.

Using the information gathered from the small group and instructor feedback sessions, students revised and iterated on their roadside marker designs within Google Blocks. The instructor provided students 2 days to further develop their promising designs using the HTC Vive and Google Blocks technologies. According to the instructor, it was at this point that students started to sculpt well in Google Blocks. He reported seeing a big jump between the initial prototype designs that students proposed and their subsequent iterations. He discussed one student's revised Google Blocks work, a design of the city's name in large limestone block letters. Originally, the letters were about 3 feet in height, but post-critique, the student enlarged the letters to 6 feet in height. Other students modified their designs to reflect more sophisticated interpretations of city symbols, as highlighted in Fig. 6.

\section{Transitioning out of 3-D VLEs}

After students revised their prototypes based on feedback, they set aside designing within 3-D VLEs for the rest of the project at the request of the instructor. The instructor now wanted his students to focus on material use and to refine their designs through a "design schematic." A limitation

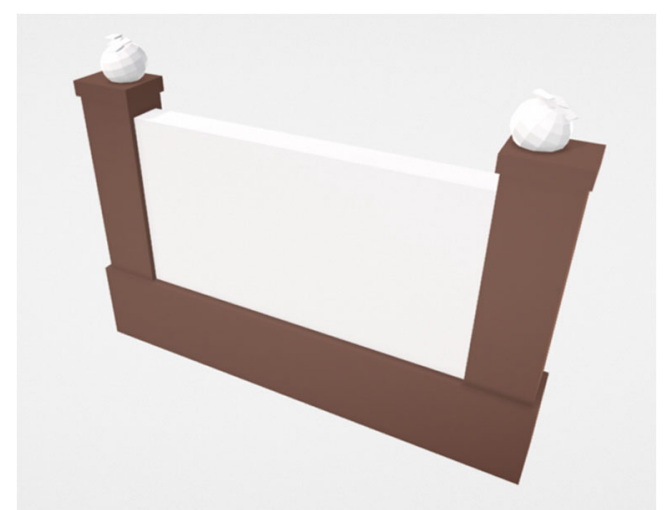

Fig. 6 A revised roadside marker design that aligned city symbols and city culture in a sophisticated manner noted earlier, Google Blocks does not provide a texture tool within the user interface, thus making material exploration difficult. To create a design schematic, students made a screen capture of their Google Block renderings and paired them with material samples that they located online. The intent of this portion of the project was to have students start to think about their designs according to material use (see Fig. 7). After schematic designs were submitted, the instructor held a large group critique in which all members of the course offered verbal feedback.

The last iteration of the project involved integrating a "pencil aesthetic" through another established 3-D modeling tool, namely, SketchUp. Google created SketchUp in 2000, so the technology has an established history and an interface in which users create 3-D models through a traditional computer software interface. The instructor wanted students to transition designs into SketchUp in order to incorporate context (i.e., realistic landscapes) into their finished roadside marker designs. To work within SketchUp, students had to export their Google Blocks designs as .obj files, which the instructor then converted to SketchUp format (.skp). (Only the premium version of SketchUp, licensed to the instructor, provided an import/ export option.) After file conversions were complete, the instructor placed the SketchUp files in Google Drive for his students to retrieve.

The last design effort in SketchUp took 1 week to complete. Since SketchUp was new to most individuals within the course, the instructor scripted what students needed to do. He created specific lecture notes, recorded tutorial videos for students to follow, and spent time in class overviewing how to

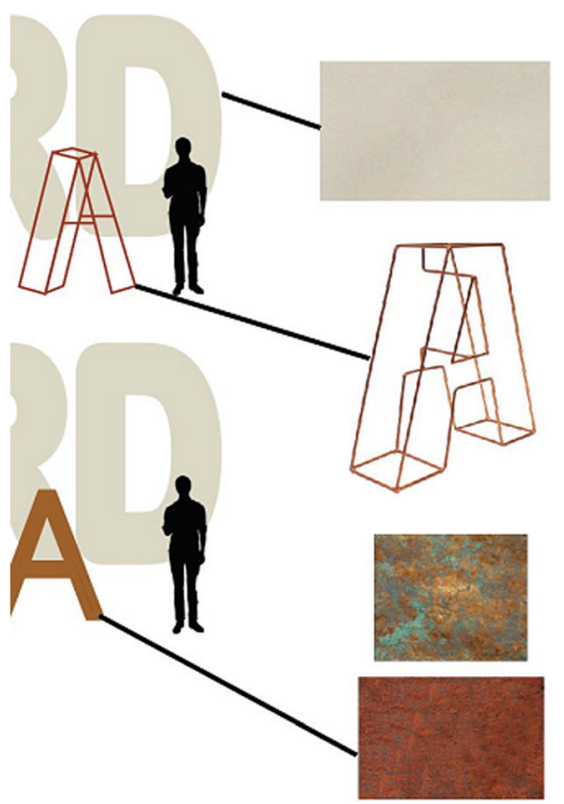

Fig. 7 Partial image of a design schematic that featured refined forms and material use 
use the product. When students went to work within SketchUp, they had the ability to incorporate the look of finished materials (e.g., limestone, rusted metal, wood) to their roadside marker designs. The instructor also directed students to use an online tool called 3-D Warehouse (https://3Dwarehouse.sketchup.com/), which offers a repository of 3D models. All students in the Design for Social Impact course used 3-D Warehouse to situate their roadside markers in context (e.g., trees, roads, landscapes) so that their designs were not floating in space. Finally, the instructor asked students to apply soft filters that were not photo realistic for an "unfinished design" look when presenting to the course's clients, the city officials. The instructor advised students that they should not present the roadside marker designs as polished, final versions to allow for potential adjustments and redesign.

\section{Sharing 3-D VLE Creations with Clients}

At the end of the project, city leaders returned to the university campus to review the students' work. Students shared their SketchUp creations with the clients and explained the cultural considerations and their design intent. Due to the large number of students in the course, city leaders were unable to ask questions or offer constructive feedback during presentations. Instead, city leaders later discussed their preferred designs at their regional offices after leaving campus.

The instructor admitted that the designs that he liked and encouraged, which tended to be "sleeker" in appearance, were not the designs that the clients ultimately preferred. The city leaders selected roadside markers that incorporated blended materials (e.g., wood, steel) with limestone. The reason for the selections by the city leaders ultimately tied back to the cultural aspects of the two cities. For example, one city possessed the oldest protected forest in the state, which brought with it visitors and economic benefits. At first, one city asked for the instructor to combine elements from different student projects, but the city ultimately ended up selecting a student's design without modifications (see Fig. 8).

\section{Additional Pedagogical Decisions Related to 3-D VLE}

Within the Design for Social Impact course, the instructor had his students work independently, rather than in groups, to provide ample opportunity to develop technical skills with Google Blocks and the other traditional modeling technologies. The instructor explained that when students are required to complete their own design work, they have increased accountability and responsibility. Additionally, given the short time frame of the eight-week project, it made sense to the instructor to ask students to design on their own and to focus group collaboration efforts on small group critiques.

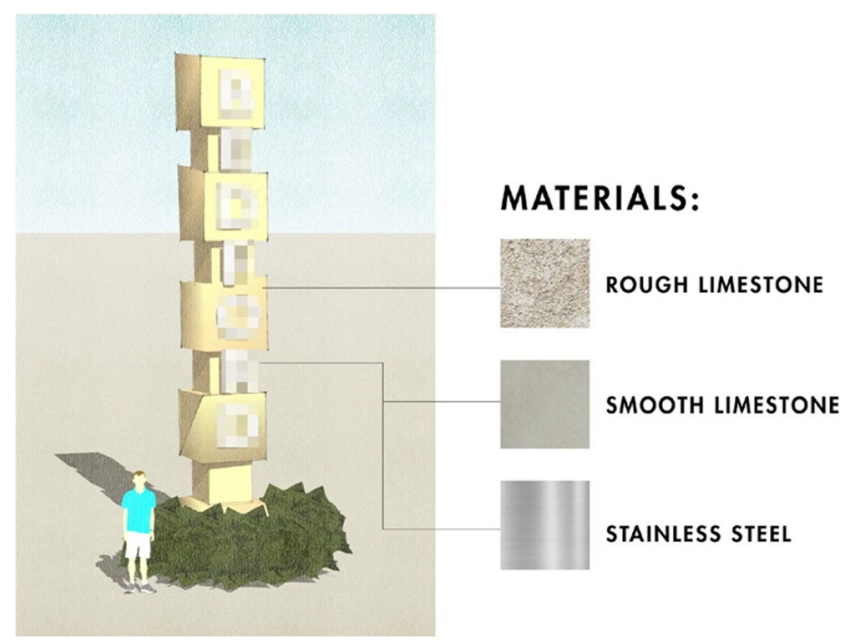

Fig. 8 The roadside marker design selected by city officials for construction in 2018. City name is blurred for purposes of anonymity

Designing collaboratively within 3-D VLEs can present challenges. Google Blocks, as a VR modeling tool, does not allow for two or more users to design collaboratively within the program. As a result, students do have the ability to create individual designs and could try to bring their creations together as a group. Determining however which 3-D VLE design should go forward can be problematic depending on the group dynamics. The instructor shared that since he did not know his students well before the course began, he was concerned about the types of group dynamics that would emerge if the students had to create roadside marker designs together. Interestingly, the instructor was informed by his Graphic Design colleague, who oversaw the first eight-week section of the course, about the students' background knowledge with design. As a result, the instructor tried to spread out the design knowledge of his class across the small feedback groups which remained constant throughout the course.

Wanting to improve the 3-D VLE experience for future students, the instructor distributed an electronic survey to his students at the end of the semester asking for feedback on their 3-D VLE experiences. Out of the 25 surveys distributed, nine surveys were completed. The 12 survey questions strictly focused on VR as a teaching tool. Student responses highlighted the logistical struggles they encountered with the VR technologies. The most common issue reported by survey respondents was that the VR motion-sensing controllers were not adequately charged, which resulted in unresponsive controllers that would shut down every few minutes. Another common problem that students cited included missing power cords for the VR technologies, as the cords could also be used to charge mobile phones. Students also reported that the HTC Vive headsets were not always clean. From an HTV Vive usage standpoint, students shared that they had a tendency to bump into tables and chairs when using the HTC Vive headsets and motion-sensing controllers in the VR computer labs on campus. 


\section{Project Significance}

In this design case, we provided detailed examples of how classroom context, regional cultures, and pedagogical aims shaped the design judgments of one particular instructor. We call on scholars who are interested in incorporating student use of 3-D VLE tools to consider the pedagogical aims of their own teaching and learning contexts, as well as the ways in which culture is embedded and aligned throughout the design process.

We also sought to demonstrate in this design case how all learners, regardless of background, can use Google Blocks to easily sketch, model, and design within 3-D VLEs. We advocate for formative peer and instructor feedback to be integrated at multiple points within the student 3-D VR modeling process. Additionally, we recognize that all 3-D VR modeling tools will have benefits and limitations; however, it is only through transparency of design judgments that we will be able to share our own 3-D VLE precedent with others. Finally, although we are encouraged by the unique learning possibilities that new 3-D VLEs afford, we ask instructors and practitioners, first and foremost, to seek out authentic learning situations where students have the opportunity to design for social impact. It is a meaningful goal, in general, to pursue.

\section{Compliance with Ethical Standards}

Ethical Approval All procedures performed in studies involving human participants were in accordance with the ethical standards of the institutional and/or national research committee and with the 1964 Helsinki declaration and its later amendments or comparable ethical standards.

Informed Consent Informed consent was obtained from all individual participants included in the study.

Conflict of Interest The authors declare that they have no conflict of interest.
Publisher's Note Springer Nature remains neutral with regard to jurisdictional claims in published maps and institutional affiliations.

\section{References}

Asino, T. I., Giacumo, L. A., \& Chen, V. (2017). Culture as a design "next": theoretical frameworks to guide new design, development, and research of learning environments. The Design Journal, 20(sup1), S875-S885.

Baepler, P., Walker, J. D., Brooks, D. C., Saichaie, K., \& Petersen, C. I. (2016). A guide to teaching in the active learning classroom: History, research, and practice. Sterling: Stylus Publishing, LLC.

Boling, E. (2010). The need for design cases: disseminating design knowledge. International Journal of Designs for Learning, $1(1), 1-8$.

Boling, E., Alangari, H., Hajdu, I. M., Guo, M., Gyabak, K., Khlaif, Z., . . . Techawitthayachinda, R. (2017). Core judgments of instructional designers in practice. Performance Improvement Quarterly, 30(3), 199-219.

Constable, H. (1994). A study of aspects of design and technology capability at key stage 1 and 2. In IDATER '94 (pp. 9-14).

Dalgarno, B., \& Lee, M. J. (2010). What are the learning affordances of 3 D virtual environments? British Journal of Educational Technology, 41(1), 10-32.

Dalsgaard, P. (2017). Instruments of inquiry: understanding the nature and role of tools in design. International Journal of Design, 11(1), 21-33.

Egan, B. (1999). Children talking about designing: How do young children perceive the functions/uses of drawing as part of the design process? In IDATER '99 (pp. 79-83).

Fowler, C. (2015). Virtual reality and learning: where is the pedagogy? British Journal of Educational Technology, 46(2), 412-422.

Howard, C. D., Boling, E., Rowland, G., \& Smith, K. M. (2012). Instructional design cases and why we need them. Educational Technology, 52(3), 34.

Lemons, G., Carberry, A., Swan, C., Jarvin, L., \& Rogers, C. (2010). The benefits of model building in teaching engineering design. Design Studies, 31(3), 288-309.

Reigeluth, C. (1999). Instructional-design theories and models (Vol. 2). Mahwah: Lawrence Erlbaum Associates. 